Reprod. Nutr. Dévelop., 1983, 23 (6), 943-958.

\title{
Digestion et absorption intestinale des phosphatildycholines
}

\author{
P. BOUCROT
}

Centre de Recherches en Biologie et en Physico-Chimie cellulaires, Faculté des Sciences 2, rue de la Houssinière, 44072 Nantes Cedex, France.

\section{Summary. Phosphatidy/cholines : digestion and intestinal absorption.}

Endogenous phosphatidylcholines from bile, desquamation of epithelial cells, bacteria, etc. are quantitatively more important than dietary phosphatidylcholines. The process of digestion and absorption of these polar lipids are still under investigation.

Pancreatic phospholipase $A_{2}$ and biliary salts must be present for phosphatidylcholine hydrolysis. The enzyme acts on the fatty acid ester bond in the 2-position of phosphatidylcholine, giving an unsaturated fatty acid and a lysophosphatidylcholine. A more complete hydrolysis of phosphatidylcholine does not occur in the intestinal lumen in man but in rats with microorganism phospholipases. In pancreatic juice, no lysophospholipase can be detected.

The extent of hydrolysis of phosphatidylcholines is still unknown. Experimental evidence in vivo indicates intense or long luminal hydrolysis of phosphatidylcholine, depending on the method used to separate the micellar and oil phases of luminal contents. Using rapid separation methods, we found low amounts of lysophosphatidylcholine in the intestinal contents. Contrary to the intense hydrolysis of the 1 and 3-position in triglycerides, which appeared using pancreatic lipase, in vivo experiments with phosphatidylcholine labelled with an unsaturated fatty acid at the 2position indicated low labelling of luminal free fatty acids.

Evidence has been found in vivo, but not in vitro, that the phosphatidylcholines of native bile were hydrolysed more slowly than dietary phosphatidylcholine. This could be explained by the existence of a lipoprotein complex in bile, containing an apoprotein, immunoglobins, phosphatidylcholine and cholesterol.

As soon as dietary and biliary phosphatidylcholines appeared in the duodenum, they were observed to be intact polar phospholipids adsorbed on the emulsion of dietary triglycerides where pancreatic lipase acts. Since phospholipase $A_{2}$ was active in the micellar phase of the intestinal contents, it is not clear why a large part of the phosphatidylcholines remained intact in the micelles. They might represent a polar material utilized by the brush borders of the enterocytes when the lipids of the mixed micelles enter.

Both phosphatidylcholine and Iysophosphatidylcholine hydrolysis occurred in the enterocytes, giving lysophosphatidylcholine and fatty acids. The lysophosphatidylcholines were either completely hydrolysed or acylated to form new phosphatidylcholine molecules, while the unsaturated fatty acids were used for triglyceride synthesis. These triglycerides and part of the phosphatidylcholines (whatever their origin) were incorporated into chylomicrons. 


\section{Introduction.}

L'alimentation humaine est plus riche en triglycérides (beurre, huile, margarine, etc...) qu'en phospholipides (PL) [surtout phosphatidylcholines (PC)] ou en cholestérol qui sont généralement associés à des protéines et apportés surtout par des aliments d'origine animale.

Les travaux relatifs à la digestion des triglycérides sont nombreux ; par contre, ceux qui concernent les phosphatidylcholines sont en nombre très limité bien que les recherches, in vitro, sur l'action de la phospholipase $A_{2}$ aient été assez développées.

Les phosphatidylcholines (origine alimentaire ou biliaire) sont peu hydrolysées dans le contenu intestinal contrairement aux triglycérides. Par leurs propriétés physiques particulières (molécules amphiphiles, action détergente, etc...) les phosphatidylcholines participent aux étapes de la digestion enzymatique des triglycérides et de l'absorption des micelles intraluminales par les entérocytes. Mais bien des résultats connus sont encore fragmentaires ou contradictoires et moins bien établis que ceux rapportés sur les rôles détergents des sels biliaires, qui ont fait l'objet de nombreux travaux.

\section{Origine des phosphatidylcholines de la lumière intestinale}

1) Exogène. - Les phosphatidylcholines ( $P C$ ) sont les constituants majeurs des PL de l'alimentation : $50 \%$ des PL du muscle et du foie de bœuf ; $30 \%$ des PL du rein et du cerveau ; les $2 / 3$ des PL du jaune d'œuf et approximativement la moitié des PL du lait. On estime la ration quotidienne de l'homme entre 0,7 et $4 \mathrm{~g}$ de $\mathrm{PC}$, principalement d'origine animale.

2) Endogène. - Des travaux anciens indiquent la présence de $\mathrm{PL}$ dans la lumière intestinale d'animaux à jeun ou soumis à des régimes exempts de $\mathrm{PL}$, mais leurs quantités n'ont été évaluées que récemment : chez le Rat (Clément et al., 1955 ; Raulin et Lefort, 1960 ; Clément, 1961 ; Di Costanzo et Clément, 1965 ; Waldram, 1975 ; Reisser, 1978) ; chez le Mouton (Zamytchkina et Grodsensky, 1955).

Ces PL endogènes proviennent :

a) de cellules: Les cellules desquamées sont nombreuses dans le tractus digestif, le renouvellement des entérocytes étant rapide ; les lipides de ces cellules sont déversés dans la lumière de l'intestin grêle : 1,12 mg/30 min (Cotton, 1971 et 1972) chez le rat à jeun ou de 12 à $30 \mathrm{~g} / \mathrm{j}$ (Croft et Cotton, 1973) pour l'appareil digestif entier chez l'Homme.

b) de sécrétions: Toutes renferment de petites quantités de lipides mais, parmi elles, la bile est la plus riche en PL $13 \mathrm{mg} / \mathrm{ml}$ de lipides totaux dont $80 \%$ de $\mathrm{PC}$ dans la bile du Rat ; 2 à $8 \mathrm{mg} / \mathrm{ml}$ dont $96 \%$ de PC dans la bile hépatique humaine) et présente un débit élevé $(9$ à $15 \mathrm{ml} / \mathrm{j}$ chez un rat de $300 \mathrm{~g}, 500$ à $1000 \mathrm{ml} / \mathrm{j}$ chez l'Homme). On détecte des traces de lysophosphatidylcholines (lyso PC : forme hydrolysée des PC) dans la bile normale, mais leur teneur aug- 
mente lorsqu'il existe des lésions de la paroi de la vésicule biliaire ou une infection des canaux biliaires.

c) de bactéries: Les microorganismes présents dans la lumière intestinale synthétisent des PL pour édifier leurs propres membranes.

Ces données chiffrées montrent que la lumière intestinale reçoit à peu près autant de $\mathrm{PL}$ endogènes que de $\mathrm{PL}$ exogènes.

\section{Phospholipase $A_{2}$ du suc pancréatique}

Les PC sont des substances amphiphiles, peu solubles dans l'eau, et peuvent être considérées comme des détergents ; en outre, elles ont la propriété de gonfler dans les milieux aqueux alors que les lyso PC sont des amphiphiles solubles.

Sur la figure 1 sont indiqués les lieux d'impact des phospholipases $A_{1}, A_{2}$, $B, C$ et D sur la molécule de phosphatidylcholine.

Dès 1948, Le Breton et Pantaléon avaient signalé l'existence d'une phospholipase dans le suc pancréatique de Chien, fait confirmé par Clément et Rigollot (1964) dans le suc pancréatique humain. Cette enzyme a été détectée, en outre, dans le suc pancréatique de Rat (Belleville et Clément, 1966a et b) ; elle est de type $A_{2}$ et se trouve sous forme de zymogène (Arnesjö et al., 1967,.

La forme zymogène est activée par action de la trypsine. L'hyclrolyse des PC se traduit par l'apparition de lysophosphatidylcholines (lyso PC) sacurés et d'acides gras insaturés. En effet, dans les-PC d'origine biologique, les acides gras saturés et insaturés sont localisés respectivement en position 1 et en position 2 sur la molécule de glycérol. La liaison ester située en position 2 est beaucoup plus labile (quelle que soit la nature de l'acide gras) que celle sitsée en position 1 (Arnesjö et al., 1969 ; Parthasarathy et al., 1974 ; Lekim et Betzing 1976).

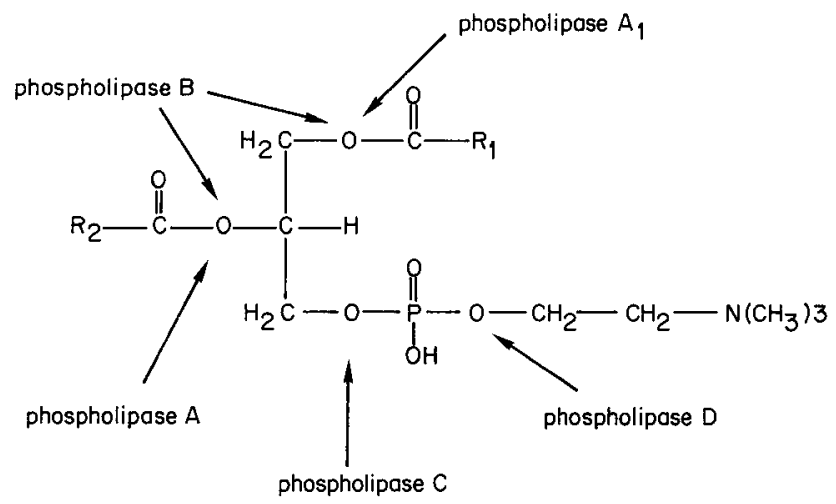

FIG. 1. - Lieu d'impact des phospholipases (A, B, C et D) sur une molécule de 1,2-diacy/-snglycéro-3-phosphocholine $(=$ lécithine $=\mathrm{PC})$. 
Il n'a pas été détecté d'activité lysophospholipase dans le suc pancréatique ni dans les contenus d'intestin grêle obtenus par aspiration chez l'homme. La phospholipase $A_{2}$ de différentes origines (venin de serpent, insectes, tissus de mammifères) est thermostable, est activée par des ions $\mathrm{Ca}^{2+}$ et les sels biliaires, en particulier le désoxycholate de sodium (Magee et al., 1962). De Haas et al. (1971) ont démontré que la phospholipase $A_{2}$ pancréatique agit principalement dans la phase micellaire du contenu intestinal.

\section{La bile}

1) Organisation micellaire des lipides biliaires. - II est généralement admis que les constituants biliaires : sels biliaires principalement, mais aussi PC et cholestérol, forment des micelles mixtes (Admirand et Small 1968 ; Montet et Dervichian, 1971). La concentration molaire des lipides est élevée : sels biliaires environ $18 \mathrm{mM}, \mathrm{PC} 3 \mathrm{mM}$ et cholestérol : 0,1 mM.

2) Complexe lipoprotéique biliaire. - Divers auteurs (Hauton et Laurent 1965 ; Lairon et al., 1972 ; Nalbone et al., 1973, 1978, 1979 ; Lafont et al. 1974, 1977 ; Manzatto et al., 1976 ; Augeire-Charbonnier et al., 1980) ont décrit, dans la bile, le complexe lipoprotéique qui comporte $P C$, cholestérol et protéines. La fraction protéique a été caractérisée : composition en acides aminés, poids moléculaire, $\mathrm{pK}$, etc... Elle est hétérogène et comprend une apolipoprotéine et plusieurs autres protéines dont les immunoglobines $\lg A$ et $\lg G$ ayant toutes une forte affinité pour les PC de la bile (Nalbone et al., 1978, 1979 ; Vigne et al., 1979, 1981).

L'existence de ce complexe est compatible avec la théorie de l'organisation micellaire des constituants biliaires. Le complexe paraît jouer un rôle dans la dispersion des lipides et la lipolyse des triglycérides, in vitro (Lairon et al., 1973, 1975, 1978a, b, c, 1980). On ignore si cette organisation macromoléculaire est préservée au moment de l'absorption des constituants micellaires. Dans l'affirmative, la bordure en brosse des entérocytes se trouverait au contact de molécules simples et de macrocomplexes.

\section{Hydrolyse des phosphatidylcholines dans la lumière intestinale}

Chez les monogastriques, il n'y a jamais de dégradation de PC avant leur arrivée dans le duodénum: Les PC exogènes sont libérées au cours d'une protéolyse qui a lieu dans I'estomac. A ce niveau, ces PC forment avec les triglycérides (lipides ingérés quantitativement les plus importants), les diglycérides et les stérols toujours en faible quantité, une émulsion "huile dans l'eau » qui se déverse par intermittence dans le duodénum.

L'hydrolyse enzymatique des PC débute dans le duodénum, sous l'effet de la phospholipase $A_{2}$ du suc pancréatique, au contact avec l'émulsion. Les sels biliaires, toujours présents à une concentration supérieure à la concentration micellaire critique, favorisent la dispersion des lipides exogènes en gouttelettes 
de plus en plus fines (Borgström, 1974) augmentant considérablement la surface de l'interphase huile-eau. De cette façon, l'hydrolyse par la lipase des triglycérides est facilitée. Les PC de bile (Kimura et al., 1972), Lairon et al., 1975, 1978a et b) ou exogènes (Kimura et al., 1972 ; Borgström, 1980) interviennent dans les phénomènes d'absorption de l'association lipase-colipase sur l'interphase triglycérides/eau. Cependant, les conditions expérimentales utilisées par ces auteurs sont très éloignées de ce qui a lieu dans la lumière intestinale et Borgström (1980) conclut que la présence de PC n'est peut-être pas obligatoire pour que la lipolyse intraluminale des triglycérides alimentaires ait lieu.

L'ampleur de l'hydrolyse intraluminale des PC fait encore l'objet de controverses. Arnesjö et al. (1969) et Parthasaruty et al. (1974) ont rapporté que dans la lumière intestinale, les quantités de lyso PC étaient toujours supérieures à celles de PC, tandis que Porter et Saunders (1971), Lairon et al. (1973), Paul et al. (1978), Boucrot (1979), Sklan et Budowski (1978) ont constaté I'inverse. Les résultats divergents peuvent provenir des conditions expérimentales, en particulier de la composition lipidique de la ration mais aussi du mode d'obtention de la phase huileuse et de la phase micellaire, isolées du contenu intestinal. Lorsque la phase micellaire est isolée par une centrifugation de $18 \mathrm{~h}$, après un chauffage à $70^{\circ} \mathrm{C}$ pendant quelques minutes, la phospholipase $A_{2}$ thermostable n'est pas dénaturée et continue d'agir sur les PC en donnant naissance à des quantités importantes de lyso PC et d'acides gras libres. Rien de tel n'apparaît si la séparation phase huileuse - phase micellaire s'effectue directement sur colonne de polyacrylamide agarose ou sur microfiltres (Porter et al., 1971 ; Porter et Saunders, 1971 ; Boucrot, 1979).

Les $\mathrm{PC}$ exogènes sont dégradées moins rapidement et séjournent plus longtemps dans la lumière intestinale que les triglycérides. En effet, il a été estimé que dans les contenus intestinaux humains, l'activité phospholipase est nettement inférieure à l'activité triglycéride lipase (Arnesjö et al., 1969).

La présence de triglycérides dans la ration, mais non de $\mathrm{PC}$ exogènes, facilite I'hydrolyse des PC biliaires (B́oquillon et Boucrot, 1980 ; Reisser et Boucrot, 1978). On peut penser que les produits issus de la lipolyse des triglycérides (monoglycérides et acides gras) modifient la taille et le nombre des micelles. En l'absence de suc pancréatique (dérivation vers l'extérieur pendant $24 \mathrm{~h}$ ) on observe une faible hydrolyse luminale des PC biliaires ou exogènes (Goux et Boucrot, 1981) : cette hydrolyse pourrait être due à des phospholipases bactériennes ou à celles de cellules intestinales desquamées.

Borgström (1957) et Arnesjö et al. (1969) pensent que, chez l'homme, l'action de la phospholipase $A_{2}$ intraluminale à l'égard des PC exogènes et des PC biliaires est identique, car ces constituants doivent former un mélange homogène dans la lumière intestinale. Pour d'autres auteurs, chez le Rat, les PC biliaires sont absorbées plus lentement que les $P C$ exogènes (Boucrot, 1972 ; Olsson et Boucrot, 1974 ; Boucrot et al., 1978 ; Reisser 1978). A l'heure actuelle, aucune explication n'a pu être fournie, l'organisation des PC biliaires dans le complexe macromoléculaire pouvant être invoquée mais sans preuve expérimentale. Par contre, in vitro, plusieurs auteurs ont accumulé un certain nombre de faits expérimentaux en faveur de l'hydrolyse préférentielle des $P C$ exogènes par 
rapport aux PC biliaires. En effet, au cours d'incubations, I'hydrolyse de PC de bile native est plus importante que dans la lumière intestinale, mais reste 3 fois plus faible que I'hydrolyse de PC exogènes (Boucrot et Clément, 1971), fait confirmé et expliqué récemment (Nalbonne et al., 1980) : dans la bile de Rat, le rapport sels biliaires/PC égale ou excède 7 , ce qui correspond à des conditions défavorables à une dégradation des $\mathrm{PC}$ puisque l'activité de la phospholipase $A_{2}$ est maximale lorsque ce rapport est voisin de 2 .

Au cours de l'incubation in vitro de bile native en présence de suc pancréatique (Boucrot et al., 1978), la classe de PC biliaires la plus rapidement hydrolysée est celle qui contient les acides gras $16: 0$ et $18: 2$ et qui est quantitativement la plus abondante (Boucrot et al. 1978; Kawamoto et al., 1980). Comme I'hydrolyse préférentielle de cette classe ne se produit plus lorsque l'on fait agir le suc pancréatique sur des PC extraites de la bile (Boucrot et al. 1978) - celles-ci ne font dès lors plus partie du complexe lipoprotéique - on doit conclure que dans la bile native, les PC sont liées au complexe lipoprotéique, et que la phospholipase $A_{2} n^{\prime} a$ pas accès au site d'hydrolyse de certaines classes de PC de bile native. Cette observation est à rapprocher de celle avancée par Uthee et Magee (1971) et Slotboom et al. (1978) selon laquelle l'action de la phospholipase $A_{2}$ sur les PL appartenant à des lipoprotéines dépend de la configuration de ces dernières.

Dans la lumière intestinale, in vivo, les conditions de dégradation des PC biliaires sont vraisemblablement différentes puisqu'on ne peut pas mettre en évidence de différences de taux d'hydrolyse entre les classes de PC biliaires (Boucrot et al., 1978).

Selon les espèces, la nature des lipides ingérés et le mode d'obtention des phases huileuse et micellaire isolées à partir du contenu intestinal, la composition des micelles varie, mais on peut retenir que la concentration maximale en acides gras est environ $10 \mathrm{mM}$, en monoglycérides $5 \mathrm{mM}$, en cholestérol exogène et endogène, $0,1 \mathrm{mM}$, en $P C$ et lyso $P C$ exogènes $1 \mathrm{mM}$ et $0,4 \mathrm{mM}$ respectivement ; par contre, les teneurs en $P C$ et lyso $P C$ biliaires 10,4 et $0,2 \mathrm{mM}$ respectivement) et en sels biliaires $(9 \mathrm{mM})$ sont plus constantes (valeurs tirées des travaux de Hofmann et Borgström, 1964 ; Miettinen et Siurala, 1971 ; Ricour et Rey, 1970 ; Porter et Saunders, 1971 ; Boucrot, 1979). Le rapport PC exogènes et endogènes/sels biliaires affecte la taille des micelles; en effet, leur diamètre augmente lorsque la valeur de ce rapport croît (Nalbone et al., 1980). Dans les micelles, la masse des lipides exogènes (monoglycérides, acides gras, cholestérol) est égale à la somme «sels biliaires + PC exogènes et endogènes + lyso PC exogènes et endogènes ». Une partie des sels biliaires sous forme de monomères n'appartiennent pas aux micelles.

\section{Absorption des phosphatidylcholines et des lysophosphatidylcholines par les entérocytes}

Elle ne peut être dissociée de l'absorption des autres constituants lipidiques des micelles mixtes du contenu intestinal. La solubilisation micellaire des lipides 
est une étape importante pour qu'ils puissent vaincre la résistance de la " couche d'eau non agitée " adjacente à l'épithélium intestinal (Wilson et Dietschy, 1972 ; Westegaard et Dietschy, 1976) et franchir la membrane plasmique des entérocytes ou bien se dissoudre dans la partie lipidique (Glickman, 1976). Une hydrolyse intraluminale des triglycérides et des phospholipides et une absorption des produits d'hydrolyse ont lieu en l'absence de sécrétion biliaire (Borgström, 1953 ; Saunders et Dawson, 1963 ; Gallagher et al., 1965 ; Blomstrand et al., 1969 ; Clouet et al., 1970 ; Goux et Boucrot, 1981). Mais des perturbations métaboliques importantes se produisent dans la muqueuse intestinale. L'estérification des fonctions alcool libres du glycérol des monoglycérides est en particulier déficiente et les acides gras non estérifiés empruntent alors la voie portale plutôt que la voie lymphatique réservée aux formes de lipides estérifiées (Saunders et Dawson, 1963 ; Knoebel et Ryan, 1965 ; Gallagher et al., 1965 ; Dietschy, 1967 ; Blomstrand et al. 1969 ; Clouet et al., 1970 ; Tandon et al., 1972 ; Rodgers, 1975).

Les $P C$ et les 1-mono-acylphosphatidylcholines d'origine exogène ou endogène sont absorbées dans le duodénum et le jéjunum chez l'Homme (Arnesjö et al., 1969) et chez le Rat (Ulrich et al., 1974 ; Reisser et al., 1980) ; par contre, chez le Mouton, elles le sont dans le jéjunum et l'iléon (Lennox et al., 1968 ; Thewis et al., 1978). Des expériences mettant en œuvre des lyso PC marquées à la fois sur le glycérol et l'acide gras (ou la choline) indiquent que ces constituants sont absorbés intacts (Parthasarathy et al., 1974 ; Nilsson et Borgström, 1967 ; Goux et Boucrot, 1980 ; Scow et al., 1967 ; Nilsson, 1968). Selon Parthasarathy et al. (1974), les lyso PC disparaissent de la lumière intestinale plus rapidement que les $P C$. D'autres auteurs montrent que des molécules de $P C$ doublement marquées, exogènes ou marquées dans de la bile native, peuvent aussi être absorbées intactes (Artom et Swanson, 1948 ; Blomstrand, 1954 ; Bloom et al., 1955 ; Hoelzl, 1972 ; Paul et al., 1978 ; Goux et Boucrot, 1980, 1981 ; Boichot et al., 1981 ; Boquillon et al., 1982).

In vitro et in vivo, l'absorption d'acide oléique et de cholestérol est diminuée après addition de bile ou de PC biliaires à des micelles mixtes (Rampone, 1972a et b), et l'addition de PC de diverses origines produit le même effet que les PC biliaires $1 O^{\prime}$ Doherthy et al., 1973 ; Rodgers et O'Connor, 1975 ; Saunders et Sillery, 1976 ; Ammon et al., 1978). Par contre, Rodgers et O'Connor (1975) observent que la présence de lyso $\mathrm{PC}$, contrairement à celle de $\mathrm{PC}$, ne modifie pas l'absorption du cholestérol et des acides gras. Pour Rampone et Long (1977) les PC ralentissent le processus d'absorption tandis que les lyso PC produisent un accroissement du taux d'absorption des acides gras et facilitent leur estérification dans la muqueuse mais n'ont aucun effet sur l'absorption du chlolestérol, ni sur son estérification. Pour Rodgers (1975), chez le Rat dont la bile est dérivée vers l'extérieur ou chez le rat témoin, les PC biliaires n'ont aucune action sur les processus d'absorption des triglycérides. Tsö et al. (1977) arrivent à la même conclusion mais seulement dans le cas où les quantités de lipides ingérés sont faibles ; dans le cas où ces dernières sont élevées, l'absorption est très ralentie ; celle-ci redevient normale après addition de PC biliaires. Ces résultats contradictoires tiennent à des conditions expérimentales souvent éloignées de celles qui 
existent dans la lumière intestinale. En effet, les concentrations molaires (1 à 4 $\mathrm{mM}$ ) de $P C$ dans les micelles sont très élevées.

Par contre, lorsque les micelles mixtes synthétiques ont une composition représentative des micelles isolées de la lumière intestinale après l'ingestion d'un repas qui a comporté des triglycérides et des $\mathrm{PC}$, la captation des acides gras par la muqueuse (sacs éversés d'intestin grêle de Rat, anse duodénojéjunale isolée, In situ, chez le rat) s'effectue à la même vitesse que celle des PC et des lyso PC d'origine exogène ou biliaire (Goux et Boucrot, 1980 ; Boichot et al., 1981 et Boichot et al., 1982 - observations non publiés). En outre, l'absence d'acides gras et de monoglycérides dans ces micelles ne modifie pas l'absorption des PC et des lyso PC micellaires (Boichot et al., 1982, - observations non publiées). Les variations de taille des micelles seraient donc limitées et cette suggestion est en accord avec les résultats récents de Rampone et Machida (1981). Ces auteurs concluent, par ailleurs, que les PC n'ont aucun effet sur l'épaisseur de la « couche d'eau non agitée " de l'épithélium intestinal.

Malgré ces observations, le rôle des PC et des lyso PC dans l'absorption des lipides n'est pas établi et reste à élucider ; en particulier, on n'est pas documenté sur la pénétration de ces molécules amphiphiles à travers la bordure en brosse des entérocytes.

\section{Métabolisme des phosphatidylcholines dans les entérocytes}

Les lipides des micelles pénètrent dans les entérocytes puis interviennent dans des sites cellulaires bien définis; pour une description détaillée, on peut consulter les articles de Sabesin (1976) et Glickman (1976). On ne sait pas exactement comment s'effectue ce transfert. Des protéines cytosoliques spécifiques s'associent peut-être à ces lipides. L'existence d'une telle protéine pour le transfert des acides gras longs jusqu'au réticulum endoplasmique lisse a été démontrée par Ockner et al. (1972). Les acides gras absorbés sont tout d'abord activés en acyl-Co $A$ dans le réticulum endoplasmique lisse puis acylent l'une des molécules suivantes : le glycérophosphate, le dihydroxyacétone phosphate, les 1- et 2-monoglycérides issus de l'hydrolyse des triglycérides, les diglycérides ou les lyso PC (voir plus loin).

Un équipement enzymatique capable d'effectuer. les réactions qui interviennent dans le métabolisme des PC a été mis en évidence dans la muqueuse intestinale, in vitro : phospholipases A et B (Schmidt et al., 1957, Epstein et Shapiro, 1959 ; Marples et Thompson, 1959 ; Robertson et Lands, 1962 ; Ottolenghi, 1964 ; Subbaiah et Ganguly, 1970a ; Bonnefis et al., 1975), lysophospholipases A1 et A2 (Epstein et Shapiro, 1959 ; Robertson, 1966 ; Bonnefis et al., 1975) lysophospholipases C et D (Wykle et al., 1977), lysolécithine-acyltranférase (Stein et Stein, 1966 ; Subbaiah et Ganguly, 1970a ; Mansbach, 1972). En 1978, Bonnefis et al. ont démontré la lipido-dépendance (lipides neutres) des phospholipases de la muqueuse intestinale de Rat. Aucune activité phospholipase C et D n'a été détectée dans la muqueuse intestinale (Bonnefis et al. 1975) de sorte que la dégradation totale des PC se ferait obligatoirement via les lyso PC. Le réticulum 
endoplasmique rugueux est le siège de la synthèse des PC. L'acylation des lyso PC a lieu dans les mitochondries et les microsomes. II est bien démontré que la synthèse de novo des PC est surtout active dans les cellules des cryptes (Johnston, 1976) ; par contre, on ignore s'il existe des niveaux privilégiés de la villosité intestinale où a lieu la resynthèse de $\mathrm{PC}$ à partir des lyso $\mathrm{PC}$.

Même si l'on admet que des molécules de PC exogènes ou biliaires sont absorbées intactes, une partie d'entre elles peut être dégradée en lyso PC dans l'entérocyte. Les lyso PC peuvent être dégradées en glycérophosphorylcholine par une lysophospholipase qui ne semble pas présenter de spécificité à l'égard de la position de l'acide gras estérifié (en position 1 ou 2 sur le glycérol). Puis la glycérophosphorylcholine est scindée en glycérophosphate et choline (Nilsson, 1968 ; Parthasarathy et al., 1974).

Les acides gras libérés sont incorporés en majeure partie dans les triglycérides de la muqueuse. Inversement, certaines molécules de lyso PC sont acylées en PC, principalement par acylation directe (Lands, 1960; Houtsmuller, 1979) mais aussi par transestérification (Erbland et Marinetti, 1965).

Selon Mansbach (1972) et Lokesh et al. (1976), seules les 1-monoacylphosphatidylcholines sont acylées; par contre, les 2-monoacylphosphatidylcholines sont toujours dégradées en totalité, bien que, selon Subbaiah et Ganguly (1970b), une enzyme permette l'acylation de la fonction alcool libre du glycérol des lyso PC, qu'elle soit localisée en position 1 ou en position 2 .

Une grande part des PC des chylomicrons lymphatiques a une origine endogène (Whyte et al., 1963), mais 20 à $30 \%$ sont synthétisées dans la muqueuse à partir des acides gras des triglycérides ingérés (Hubscher et al., 1964 ; Boucrot et Clément 1965 ; Arvidson et Nilsson, 1972). $40 \%$ des PC lymphatiques ont pour origine les PC exogènes (Scow et al., 1967), 50 \% pour Fox et al. (1979), + de $50 \%$, chez l'Homme, fixées davantage dans les lipoprotéines de faible densité que dans les chylomicrons (Beil et Grundy, 1980). Cependant, après infusion de quantités élevées de PC dans l'intestin grêle, la totalité des PC des chylomicrons aurait pour unique origine les PC infusés (Mansbach, 1977 ; Mansbach et al., 1979) ; dans ce cas, la synthèse de novo de $P C$ dans la muqueuse intestinale aurait été inhibée. II existe peut-être plusieurs " pools " de PC. Selon un article récent de Steere et Mansbach (1980), en l'absence de PC dans le duodénum, les lyso $\mathrm{PC}$ des lipoprotéines sériques contribuent à la synthèse des $\mathrm{PC}$ des chylomicrons $(17 \%)$.

\section{Excrétion des phospholipides}

II n'y a pas d'absorption de PL dans le gros intestin ; les PL trouvés dans la lumière du gros intestin appartiennent aux cellules bactériennes. Chez l'Homme, les PL fécaux ont pour principale origine les membranes des bactéries excrétées avec les fèces. Chez les animaux coprophages et chez les ruminants, ces bactéries sont abondantes dès l'estomac ou le rumen. 
L'excrétion fécale quotidienne de PL chez l'Homme varierait selon la teneur et la nature des lipides du régime : elle varierait de 53 à $200 \mathrm{mg}$ après administration d'un régime lipidoprive (Ali et Kuksis, 1967a) ; elle serait de $400 \mathrm{mg}$ lorsque l'apport lipidique du régime représente 35 à $60 \%$ des calories (Ali et Kuksis, 1967b). Selon Ali et Kuksis (1967a) les phosphatidyléthanolamines sont les PL dominants des fèces; les phosphatidylinositols et les phosphatidylglycérols sont bien représentés, tandis que les PC sont en très faibles proportions. L'administration de bactériostatiques à des rats diminue les proportions de lysophospholipides dans les fèces (Raulin et Lefort, 1960).

\section{Conclusion}

Contrairement aux triglycérides alimentaires qui sont hydrolysés par la lipase pancréatique avant d'être absorbés, les travaux les plus récents montrent que, in vivo, dans la lumière intestinale, la phospholipase $A_{2}$ pancréatique hydrolyse peu les phosphatidylcholines. Dans les micelles luminales, on trouve donc à la fois des phosphatidylcholines et des lysophosphatidylcholines qui sont absorbées sous cette forme.

Par contre, in vitro, les phosphatidylcholines isolées sont hydrolysées par la phospholipase $A_{2}$. Elles le sont plus aisément que les phosphatidylcholines de bile native. Ce fait peut être expliqué par l'organisation des lipides biliaires dans un complexe constitué d'une apoprotéine, d'immunoglobines, de phosphatidylcholines et de cholestérol.

Dans les entérocytes, une fraction des phosphatidylcholines absorbées intactes sont alors hydrolysées totalement ou jusqu'à la forme lysophosphatidylcholines. Ces dernières s'ajoutent aux lysophosphatidylcholines apparues dans la lumière intestinale et peuvent subir soit une dégradation totale, soit donner de nouvelles molécules de phosphatidylcholines. Une partie des phosphatidylcholines participe à la formation des chylomicrons lympathiques.

On ne connaît pas encore le rôle que peuvent avoir les phosphatidyicholines au cours de la digestion des lipides, ni ce qui les distingue des lysophosphatidylcholines : intervention au cours du transfert des constituants lipidiques micellaires à travers l'épithélium intestinal, etc...

Reçu en février 1983. Accepté en juin 1983.

\section{Références}

ADMIRAND W. H., SMALL D. M., 1968. The physicochemical basis of cholesterol gallstone formation in man. J. clin. Invest., 47, 1043.

ALI S. S., KUKSIS A., 1967a. Excretion of phospholipids by men on fat-free diet. Can. J. Biochem., 45, 689-702. 
ALI S. S., KUKSIS A., 1967b. Excretion of phospholipids by men on high fat diets. Can. J. Biochem., 45, 703-714.

AMMON H. V., THOMAS P. J., PHILLIPIS S. F., 1978. Effect of lecithin on jejunal absorption of micellar lipids in man and on their monomer activity in vitro. Lipids, 14, 395-400.

ARNESJÖ B., BARROWMAN J., BORGSTRÖM B., 1967. The zymogen of phospholipase $A_{2}$ in rat pancreatic juice. Acta chem. scand., 21, 2897-2900.

ARNESJÖ B., NILSSON A., BARROWMAN J., BORGSTRÖM B., 1969. Intestinal digestion and absorption of cholesterol and lecithin in the human. Scand. J. Gastroenterol., 4, 653-665.

ARTOM C., SWANSON M. A., 1948. On the absorption of phospholipids. J. biol. Chem., 175, 871881.

ARVIDSON G. A. E., NILSSON A., 1972. Formation of lymph chylomicron phosphatidylcholines in the rat during the absorption of safflower oil or triolein. Lipids, 7, 344-348.

AUGEIRE-CHARBONNIER M., VIGNE J. L., NALBONE G., LAIRON D., LĖONARDI J., DOMINGO N., LAFONT H., HAUTON J. C., 1980. Effect to the polyanions precipitation on the structure of the bile lipoprotein complex. Biochimie, 62, 277-281.

BEIL F. U., GRUNDY S. M., 1980. Studies on plasma lipoproteins during absorption of exogenous lecithin in man. J. Lipid Res., 21, 525-536.

BELLEVILLE J., CLÉMENT J., 1966a. Mise en évidence d'une phospholipase A dans le suc pancréatique d'homme et de rat. Bull. Soc. Chim. biol., 48, 186-189.

BELLEVILLE J., CLÉMENT J., 1966b. Mise en évidence d'une phospholipase A dans le suc pancréatique d'Homme et de Rat. Arch. Sci. physiol., 20, 249-262.

BLOMSTRAND R., 1954. On the intestinal absorption of phospholipids in the rat. Acta chem. scand., 8, 1945-1946.

BLOMSTRAND R., CARLBERGER G., FORSGREN L., 1969. Intestinal absorption and metabolism of ${ }^{14} \mathrm{C}$-labelled fatty acids in the absence of bile in man. Acta chir. Scand., 135, 329339.

BLOOM B., KIYASU J. Y., REINHARDT W. O., CHAIKOFF I. L., 1955. Absorption of phospholipids. Manner of transport from intestinal lumen to lacteals. Am. J. Physiol., 177, 84-86.

BOICHOT J., GOUX M., BOUCROT P., 1981. Micellar phospholipids and oleic acid uptake by rat intestinal sacs, in vitro. Biochimie, 63, 445-449.

BONNEFIS M. J., LLOVERAS J., DOUSTE-BLAZY L., 1975. Spécificité d'action des phospholipases de la muqueuse intestinale du Rat. C. R. Acad. Sci. Paris, Sér. D, 280, 661-664.

BONNEFIS M. J., MARMOUYET J., REY G., THOUVENOT J. P., DOUSTE-BLAZY L. 1978. Influence des lipides de la muqueuse intestinale de rat sur son activité phospholipasique. Biochimie, 60, 521-524.

BOQUILLON M., BOICHOT J., CHABIN M., BOUCROT P., 1982. Absorption de dipalmitoyl et de distéaoryl-phosphatidylcholines micellaires par une anse intestinale chez le rat. Biochimie, 64, 65-68.

BOQUILLON M., BOUCROT P., 1980. Influence de l'apport et du mode d'administration de triglycérides sur l'absorption et l'hydrolyse des phosphatidylcholines de bile chez le Rat. Reprod. Nutr. Dévelop., 20, 1309-1316.

BORGSTRÖM B., 1953. The effect of bile diversion on fat absorption in the rat. Acta physiol. scand., 28, 279-286.

BORGSTRÖM B., 1957. Studies of the phospholipids of human bile and small intestinal contents. Acta chem. scand., 11, 749-751.

BORGSTRÖM B., 1974. Fat digestion and absorption, 555-620. In SMITH D. H., Biomembranes, 4B, Intestinal absorption. Plenum Press, London and New York.

BORGSTRÖM B., 1980. Importance of phospholipids, pancreatic phospholipase $A_{2}$ and fatty acid for the digestion of dietary fat. In vitro experiments with the porcine enzymes. Gastroenterology, 78, 954-962.

BOUCROT P., 1972. Is there an entero-hepatic circulation of the bile phospholipids? Lipids, 7, 282-288.

BOUCROT P., 1979. Prépondérance des phosphatidylcholines par rapport aux lysophosphatidylcholines dans les micelles de l'intestin de rat. J. Physiol. Paris, 75, 911-918. 
BOUCROT P., CLÉMENT J., 1965. Apport d'acides gras endogènes dans la lymphe chez le Rat après administration d'un repas contenant des triglycérides mixtes stéarique ${ }^{14} \mathrm{C}$ - oléique ${ }^{3}$ H. C. R. Acad. Sci. Paris, Sér. D., 260, 4083-4086.

BOUCROT P., CLÉMENT J. R., 1971. Resistance to the effect of phospholipase $A_{2}$ of the biliary phospholipids during incubation of bile. Lipids, 6, 652-656.

BOUCROT P., REISSER D., CLÉMENT J., 1978. The behavior of rat bile phospholipids in the intestine and in incubation media containing pancreatic juice. Lipids, 13, 791-795.

CLÉMENT G., CLÉMENT J., DREVET M., 1955. Composition des lipides du contenu intestinal après ingestion d'huile; influence du suc pancréatique et de la bile. J. Physiol. Paris, 47, 136-137.

CLÉMENT J., 1961. Les lipides intestinaux et fécaux chez le Rat soumis pendant de longues périodes à un régime lipidoprive. Arch. Sci. physiol., 15, 345-361.

CLÉMENT J., RIGOLLOT B., 1964. Etude in vitro de quelques activités enzymatiques (lipase, cholestérolestérase et phosphatidase) des sucs pancréatique et duodénal humains. Nutr. Dieta, 6. 61-75.

CLOUET E., PARIS R., CLÉMENT J., 1970. Absorption et sort de l'acide oléique ingéré chez le rat porteur d'une fistule biliaire. Arch. Sci. physiol., 24, 297-306.

COTTON P. B., 1971. The contribution of the mucosa to non-dietary lipid in the intestinal lumen. M. D. Thesis, Cambridge.

COTTON P. B., 1972. Non-dietary lipid in the intestinal lumen. Gut, 13, 675-681.

CROFT D. N., COTTON P. B., 1973. Gastro-intestinal cell loss in man; its measurement and significance. Digestion, 8, 144-160.

DE HAAS G. H., BONSEN P. P. M., PIETERSON W. A., VAN DEENEN L. L. M., 1971. Studies on phospholipase $A$ and its zymogen from porcine pancreas: III. Action of the enzyme on short-chain lecithins. Biochim. biophys. acta, 239, 252-266.

DE HAAS G. H., SARDA L., ROGER J., 1965. Positional specific hydrolysis of phospholipids by pancreatic lipase. Biochim. biophys. Acta, 106, 638-640.

DI COSTANZO G., CLĖMENT J., 1965. Incorporation in vivo des acides oléique, stéarique et palmitique tritiés dans les lipides de la lumière et de la muqueuse intestinale chez le Rat. Importance des acides gras endogènes. Bull. Soc. Chim. biol., 47, 57-68.

DIETSCHY J. M., 1967. Effects of bile salts on intermediate metabolism of the intestinal mucosa. Fed. Proc., 26, 1589.

EPSTEIN B., SHAPIRO B., 1959. Lecithinase and lysolecithinase in intestinal mucosa. Biochem. J., 71, 615-619.

ERBLAND J. F., MARINETTI G. V., 1965. The enzymatic acylation and hydrolysis of lysolecithin. Biochim. biophys. Acta, 106, 128-138.

FOX J. M., BETZING H., LEKIM D., 1979. Pharmacokinetics of orally ingested phosphatidylcholine. Nutrition and the brain, 5, 95-108.

GALLACHER N., WEBB J., DAWSON A. M., 1965. The absorption of ${ }^{14} \mathrm{C}$ oleic acid and ${ }^{14} \mathrm{C}$ triolein in bile fistula rat. Clin. Sci., 29, 73-82.

GLICKMAN R. M., 1976. Chylomicron formation by the intestine, 99-110. In ROMMEL K., GOEBELL H., BOHMER R., Lipid absorption : biochemical and clinical aspects. MTP Press Ltd, St Leonard's House, Lancaster, Grande-Bretagne.

GOUX M., BOUCROT P., 1980. Absorption de phosphatidylcholines et de lysophosphatidylcholines micellaires doublement marquées par des sacs éversés d'intestin de rat. C. R. Acad. Sci. Paris, sér. D, 290, 1015-1018.

GOUX M., BOUCROT P., 1981. Absorption intestinale de phosphatidylcholines chez le Rat après dérivation du suc pancréatique et de la bile. J. Physiol. Paris, $77,675-681$.

HAUTON J. C., LAURENT B., 1965. Un " centre de cohésion " en partie peptidique est-il nécessaire au transport des lipides? Bull. Soc. Chim. biol., 47, 2175-2177.

HOELZL J., 1972. Studies on the excretion of a ${ }^{14} \mathrm{C}{ }^{32} \mathrm{p}$ polyene phosphatidylcholine in the bile, 81-82. In GANGULY and SMELLIE, Current trends in the biochemistry of lipids. Acad. Press, New York.

HOFMANN A. F., BORGSTRÖM B., 1964. The intraluminal phase of fat digestion in man : the lipid content of the micellar and oil phases of intestinal content obtained during fat digestion and absorption. J. clin. Invest., 43, 247-257. 
HÜBSCHER G., SMITH M. E., GURR M. I., 1964, 229-242. In R.M.C. DAWSON, D. N. RHODES, Metabolism and physiological significance of lipids, John Wiley and Sons Inc., New York.

JOHNSTON H. M., 1976. Triglyceride biosynthesis in the intestinal mucosa, 85-98. ROMMEL K., GOEBELL H., BOHMER R., Lipid absorption : biochemical and clinical aspects. MTP Press Ltd, St Leonard's House, Lancaster, Grande-Bretagne.

HOUTSMULLER U.M.T., 1979. Metabolic fate of dietary lecithin. Nutrition and the brain, 5, 83-94.

KAWAMOTO T., AKINO T., NAKAMURA M., MORI M., 1980. Metabolism of individual molecular species of phosphatidylcholine in the liver subcellular membranes and bile. Biochim. biophys. acta, 619, 35-47.

KIMURA H., KITAMURA T., TSUJI M., 1972. Studies on human pancreatic lipase. I. Interconversion between low and high molecular forms of human pancreatic lipase. Biochim. biophys. acta, 270, 307-316.

KNOEBEL L. K., RYAN J. M., 1965. Effect of exclusion of pancreatic juice on digestion and mucosal absorption of fat in dogs. Proc. Soc. exp. Biol. Med., 118, 161-167.

LAFONT H., LAIRON D., DOMINGO G., NALBONE G., HAUTON J. C., 1974. Does a lecithinpolypeptide association in bile originate from membrane structural subunits? Biochimie, 56, 465-468.

LAFONT H., NALBONE G., LAIRON D., DAGORN J. C., DOMINGO N., AMIC J., HAUTON J. C., 1977. Zone electrophoresis study of the bile lipoprotein complex. Biochimie, 59, 445-452.

LAIRON D., CHARBONNIER-AUGEIRE M., NALBONE G., LEONARDI J., HAUTON J. C., PIERONI G., FERRATO F., VERGER R., 1980. The influence of bile salts and bile lipoprotein complex on pancreatic lipase hydrolysis of monomolecular films. Biochim. biophys. Acta, 618, 106-118.

LAIRON D., LAFONT H., DOMINGO N., NALBONE G., HAUTON J., 1973. On the state of lipids in the rat small intestine after a fatty meal. Biochimie, 55, 1165-1166.

LAIRON D., LAFONT H., HAUTON J. C., 1972. Lack of mixed micelles bile salt-lecithincholesterol in bile and presence of a lipoproteic complex. Biochimie, 54, 529-530.

LAIRON D., NALBONE G., DOMINGO N., LAFONT H., JULIEN R., RATHELOT J., CANIONI P., SARDA L., 1975. In vitro studies on interaction of rat pancreatic lipase and colipase with biliary lipids. Lipids, 10, 262-265.

LAIRON D., NALBONE G., LAFONT H., DOMINGO N., HAUTON J. C., 1978a. Protective effect of biliary lipids on rat pancreatic lipase and colipase. Lipids, 13, 211-216.

LAIRON D., NALBONE G., LAFONT H., LEONARDI J., DOMINGO N., HAUTON J. C., VERGER R., 1978b. Inhibition of lipase absorption at interfaces. Roles of bile salt micelles and colipase. Biochemistry, 17, 205-208.

LAIRON D., NALBONE G., LAFONT H., LEONARDI J., DOMINGO N., HAUTON J. C., VERGER R., 1978c. Possibles roles of bile lipids and colipase in lipase absorption. Biochemistry, 17, 5263-5269.

LANDS W. E. M., 1960. Metabolism of glycerolipids. II. The enzymatic acylation of lysolecithin. J. biol. Chem., 235, 2233-2237.

LE BRETON E., PANTALÉON J., 1948. Mise en évidence d'une cholestérolestérase et d'une lécithase dans le suc pancréatique, leur rôle. Arch. Sci. physiol., 2, 81-90.

LEKIM D., BETZING H., 1976. Intestinal absorption of polyunsaturated phosphatidylcholine in the rat. Hoppe-Seyler's Z. Physiol. Chem., 357, 1321-1331.

LENNOX A. M., LOUGH A. K., GARTON G. A., 1968. Observations on the nature and origin of lipids in the small intestine of the sheep. Br. J. Nutr., 22, 237-246.

LOKESH B. R., RAO A. M., MURTHY S. K., 1976. Studies on acylation of lysolecithin in chicken intestine. Indian J. Biochem. Biophys., 13, 371-375.

MAGEE W. L., GALLAI-HATCHARD J., SANDERS H., THOMPSON R. H. S., 1962. The purification and properties of phospholipase A from human pancreas. Biochem. $J$., 83, 17-23.

MANSBACH C. M., 1972. Lysolecithin acyltransferase in hamster intestinal mucosa. Lipids, 7 , 593-595. 
MANSBACH C. M., 1977. The origin of chylomicron phosphatidylcholine in the rat. $J$. clin. Invest., 60, 411-420.

MANSBACH C. M., PARTHASARATHY S., 1979. Regulation of de novo phosphatidylcholine synthesis in rat intestine. J. biol. Chem., 254, 9688-9694.

MANZATTO E., FELLIN R., BAGGIO G., WALCH S., NEUBECK W., SEIDEL D., 1976. Formation of lipoprotein $X$ : its relations to bile compounds. J. clin. Invest., 57, 1248-1260.

MARPLES E. A., THOMPSON R. H. S., 1959. The distribution of phospholipase B in mammalian tissues. Biochem. J., 74, 123-127.

MIETTINEN T. A., SIURALA M., 1971. Bile salts, sterols, sterol esters, glycerides and fatty acids in micellar and oil phases of intestinal contents during fat digestion in man. Z. Klin. Chem. Klin. Biochem., 9, 47-52.

MONTET J. C., DERVICHIAN D. G., 1971. Solubilisation micellaire du cholestérol par les sels biliaires et les lécithines extraits de la bile humaine. Biochimie, 53, 751-754.

NALBONE G., LAFONT H., DOMINGO N., LAIRON D., PAUTRAT G., HAUTON J., 1973. Ultramicroscopic study of the bile lipoprotein complex. Biochimie, 55, 1503-1506.

NALBONE G., LAFONT H., LAIRON D., VIGNE J. L., DOMINGO N., LEONARDI J., HAUTON J. C., 1978. Immunogenicity of the apoprotein of the bile lipoprotein complex. Biochimie, 60, 691-694.

NALBONE G., LAFONT H., VIGNE J. L., DOMINGO N., LAIRON D., CHABERT C., LECHENE P., HAUTON J. C., 1979 The apoprotein fraction of the bile lipoprotein complex: isolation, partial characterization and phospholipid binding properties. Biochimie, 61, 1029-1041.

NALBONE G., LAIRON D., CHARBONNIER-AUgeIRE M., VIGNE J. L., LEONARDI J., CHABERT C., HAUTON J. C., VERGER R., 1980. Pancreatic phospholipase $A_{2}$ hydro lysis of phosphatidylcholines in various physico-chemical states. Biochim. biophys. Acta, 620, 612-625.

NILSSON A., 1968. Intestinal absorption of lecithin and lysolecithin by lymph fistula rats. Biochim. biophys. Acta, 152, 379-390.

NILSSON A., BORGSTRÖM B., 1967. Absorption and metabolism of lecithin and lysolecithin by small intestinal slices. Biochim. biophys. Acta, 137, 240-254.

OCKNER R. K., MANNING J. M., POPPENHAUSEN R. B., HO W. K., 1972. A binding protein for fatty acids in cytosol of intestinal mucosa, liver, myocardium and other tissues. Science, 177, 56-58.

O'DOHERTHY P. J. A., KAKIS G., KUKSIS A., 1973. Role of luminal lecithin in intestinal fat absorption. Lipids, 8, 249-255.

OLSSON O., BOUCROT P., 1974. Résistance des phospholipides endogènes intraluminaires à I'hydrolyse pancréatique chez le Rat intact. Arch. int. Physiol. Biochim., 82, 679-699.

OTTOLENGHI A., 1964. Estimation and subcellular distribution of lecithinase activity in rat intestinal mucosa. J. Lipid Res., 5, 532-537.

PARTHASARATHY S., SUBBAIAH P. V., GANGULY J., 1974. The mechanism of intestinal absorption of phosphatidylcholine in rats. Biochem. J., 140, 503-508.

PAUL R., RAMESHA C. S., PARTHASARATHY S., GANGULY J., 1978. Effect of dietary lipids on the secretion of biliary lipids in rats. Ind. J. Biochim. Biophys., 15, 401-406.

PORTER H. P., SAUNDERS D. R., 1971. Isolation of the aqueous phase of human intestinal contents during the digestion of a fatty meal. Gastroenterology, 60, 997-1007.

PORTER H. P., SAUNDERS D. R., TYTGAT G., BRUNSER O., RUBIN C. E., 1971. Fat absorption in bile fistula man. A morphological and biochemical study. Gastroenterology, 60, 1008-1019.

RAMPONE A. J., 1972a. The effects of bile salt and raw bile on the intestinal absorption of micellar fatty acid in the rat, in vitro. J. Physiol. London, 222, 679-690.

RAMPONE A. J., 1972b. Bile salt and non-bile salt components in bile affecting micellar cholesterol uptake by rat intestine in vitro. J. Physiol. London, 227, 889-898.

RAMPONE A. J., LONG L. R., 1977. The effect of phosphatidylcholine and lysophosphatidylcholine on the absorption and mucosal metabolism of oleic acid and cholesterol in vitro. Biochim. biophys. Acta, 486, 500-510. 
RAMPONE A. J., MACHIDA C. M., 1981. Mode of action of lecithin in suppressing cholesterol absorption. J. Lipid Res., 22, 744-752.

RAULIN J., LEFORT D., 1960. Importance et caractéristiques du complexe phospholipidique intraluminaire étudié chez le Rat. Influence du régime aliméntaire et des bactériostatiques. Arch. Sci. physiol., 14, 239-255.

REISSER D., 1978. Absorption des phosphatidylcholines de bile native doublement marquées (glycérol ${ }^{3} \mathrm{H}$-acide linoléique ${ }^{14} \mathrm{C}$ ). C. R. Acad. Sci. Paris, Sér. D., 287, 733-736.

REISSER D., BOQUILLON M., CLÉMENT J., 1980. Comparison between cholesterol and phosphatidylcholine intestinal absorption from native bile labelled with $\left[1-2-{ }^{3} \mathrm{H}\right]$ cholesterol and $[1-14 \mathrm{C}]$ palmitic acid in the rat. J. Physiol. Paris, 76, 865-870.

REISSER D., BOUCROT P., 1978. In vitro and in vivo effects of exogenous lipids on the enzymatic hydrolysis of rat bile phospholipids. Lipids, 13, 796-800.

RICOUR C., REY J., 1970. Study of the oil and micellar phase during fat digestion in the normal child. Rev. eur. Etud. clin. biol., XV, 287-292.

ROBERTSON A. F., 1966. Lecithinase and lysolecithinase activity in rat tissues. Biochim. biophys. Acta, 116, 379-381.

ROBERTSON A. F., LANDS W. E. M., 1962. Positional specificities in phospholipid hydrolyses. Biochemistry, 1, 804-810.

RODGERS J. B., 1975. Lipid absorption in bile fistula rats. Lack of a requirement for biliary lecithin. Biochim. biophys. Acta, 398, 92-100.

RODGERS J. B., O'CONNOR P. J., 1975. Effect of phosphatidylcholine on fatty acid and cholesterol absorption from mixed micellar solutions. Biochim. biophys. Acta, 409, 192-200.

SABESIN S. M., 1976. Ultrastructural aspects of the intracellular assembly, transport and exocytosis of chylomicrons by rat intestinal absorptive cells, 113-145. In ROMMEL K., GOEBELL H., Lipid absorption : biochemical and clinical aspects. MTP Press Ltd, St Leonard's House, Lancaster, England.

SAUNDERS D. R., DAWSON A. M., 1963. The absorption of oleic acid in the bile fistula rat. Gut, 4, 254-260.

SAUNDERS D. R., SILLERY J., 1976. Lecithin inhibits fatty acid and bile salt absorption from small intestine in vivo. Lipids, 11, 830-832.

SCHMIDT G., BESSMAN M. J., THANNHAUSER S. J., 1957. Enzymic hydrolysis of cephalin in rat intestinal mucosa. Biochim. biophys. Acta, 23, 127-138.

SCOW R. O., STEIN Y., STEIN O., 1967. Incorporation of dietary lecithin and lysolecithin into lymph chylomicrons in the rat. J. biol. Chem., 242, 4919-4924.

SKLAN D., BUDOWSKI P.,1978. Hydrolysis of biliary phospholipids in the upper small intestine of the chick. Lipids, 13, 158-160.

SLOTBOOM A. J., Van DAM-MIERAS M. C. E., JANSEN E. H. J. M., PATTUS F., VERHEIJ H. M., De HAAS G. H., 1978. Relationship between structure and activity of pancreatic phospholipase $A_{2}$. In Enzymes of lipid metabolism, S. GATT, L. FREYSZ, P. MANDEL, Adv. exper. Med. Biol., vol. 101, Plenum Press, New York, London.

STEERE M., MANSBACH C. M., 1980. The contribution of serum phosphatidylcholine and lysophosphatidylcholine to lymph phosphatidylcholine. Biochim. biophys. Acta, 620, 462-471.

STEIN Y., STEIN O., 1966. Metabolism of labelled lysolecithin, lysophosphatidyl-ethanolamine and lecithin in the rat. Biochim. biophys. Acta, 116, 95-107.

SUBBAIAH P. V., GANGULY J., 1970a. Studies on the phospholipases of rat intestinal mucosa. Biochem. J., 118, 233-239.

SUBBAIAH P. V., GANGULY J., 1970b. Acylation of lysolecithin in the intestinal mucosa of rats. Biochem. J., 118, 241-246.

TANDON R., EDMONDS R. H., RODGERS J. B., 1972. Effects of bile diversion on the lipidreesterifying capacity of the rat small bowel. Gastroenterology, 63, 990-1003.

THEWIS A., FRANÇOIS E., THIELEMANS M. F., 1978. Etude quantitative de l'absorption et de la sécrétion du phosphore total et du phosphore phospholipidique dans le tube digestif de Mouton. Ann. Biol. anim. Biochim. Biophys., 18, 1181-1195. 
TSÖ B., BALINT J. A., SIMMONDS W. J., 1977. Role of biliary lecithin in lymphatic transport of fat. Gastroenterology, 73, 1362-1367.

ULRICH C., BOUCROT P., CLÉMENT J., 1974. Site of biliary phospholipid absorption in the small intestine of the rat. Biochimie, 56, 429-434.

UTHEE J. F., MAGEE W. L., 1971. Phospholipase $A_{2}$ : Comparative action of three different enzyme preparations on selected lipoprotein systems. Can. J. Biochem., 49, 785-794.

VIGNE J. L., LAFONT H., NALBONE G., DOMINGO N., CHARBONNIER-AUGEIRE M., LAIRON D., LEONARDI J., HAUTON J. C., 1979. Immunological relationship between bile lipoprotein complex and high density lipoprotein. Biochem. Biophys. Res. Com., 88, 1284-1287.

VIGNE J. L., NALBONE G., LAFONT H., LAIRON D., CHABERT C., DOMINGO N., HAUTON J. C., 1981. Interaction of immunoglobulins and lipids in human gallblader bile. Biochimie, 63, 735-742.

WALDRAM R., 1975. Mechanisms of lipid loss from the small intestinal mucosa. Gut, 16, 118-124.

WESTEGAARD H., DIETSCHY J. M., 1976. The mechanism whereby bile acid micelles increase the rate of fatty acid and cholesterol uptake into the intestinal mucosal cell. J. clin. Invest., 58, 97-108.

WHYTE H. M., KARMEN A., GOUDMAN D. S., 1963. Fatty acid esterification and chylomicron formation during fat absorption. 2. Phospholipids. J. Lipid Res., 4, 322-329.

WILSON F. A., DIETSCHY J. M., 1972. Characterization of bile acid absorption across the unstirred water layer and brush border of the rat jejunum. J. clin. Invest., 51, 3015-3026.

WYKLE R. L., KRAEMER W. F., SCHREMMER J. M., 1977. Studies of lysophospholipase D of rat liver and other tissues. Arch. Biochem. Biophys., 184, 149-155.

ZAMYTCHKINA K. S., GRODSENŚKY D. E., 1955. The role of radioactive isotopes in investigating the physiology and biochemistry of digestion. Proc. int. Conf. on the Peacef. Uses of Atomic Energy, Geneva, 12, 257-265. 